\title{
Médiévales
}

Langues, Textes, Histoire

79 | automne 2020

Éthiopie, Nubie, Égypte

Sharon Farmer, The Silk Industries of Medieval Paris. Artisanal Migration, Technological Innovation, and Gendered Experience

Philadelphie (PA), University of Pennsylvania Press (« The Middle Ages

Series »), 2017, $354 \mathrm{p}$.

\section{Boris Bove}

\section{OpenEdition}

\section{Journals}

Édition électronique

URL : https://journals.openedition.org/medievales/11147

DOI : 10.4000/medievales. 11147

ISSN : 1777-5892

Éditeur

Presses universitaires de Vincennes

Édition imprimée

Date de publication : 28 décembre 2020

Pagination : 225-228

ISBN : 978-2-37924-146-8

ISSN : 0751-2708

Référence électronique

Boris Bove, "Sharon Farmer, The Silk Industries of Medieval Paris. Artisanal Migration, Technological Innovation, and Gendered Experience », Médiévales [En ligne], 79 | automne 2020, mis en ligne le 28 janvier 2021, consulté le 03 janvier 2023. URL : http://journals.openedition.org/medievales/11147 DOI : https://doi.org/10.4000/medievales. 11147

Ce document a été généré automatiquement le 3 janvier 2023

Tous droits réservés 


\section{Sharon Farmer, The Silk Industries of Medieval Paris. Artisanal Migration, Technological Innovation, and Gendered Experience}

Philadelphie (PA), University of Pennsylvania Press (« The Middle Ages Series »), 2017, $354 \mathrm{p}$.

\section{Boris Bove}

\section{RÉFÉRENCE}

Sharon Farmer, The Silk Industries of Medieval Paris. Artisanal Migration, Technological Innovation, and Gendered Experience, Philadelphie (PA), University of Pennsylvania Press («The Middle Ages Series »), 2017, 354 p.

1 Dans son dernier livre, Sharon Farmer se propose d'étudier la filière de la soie à Paris comme un objet total en articulant ses dimensions géographiques, techniques, corporatives, urbaines, genrées et financières. Cela implique une mise en contexte très large qui la conduit à écrire, à partir de sources normatives, fiscales, comptables et judiciaires, une véritable histoire économique et sociale de Paris aux $\mathrm{XIII}^{\mathrm{e}}$ et $\mathrm{XIV}^{\mathrm{e}}$ siècles. Elle apporte aussi beaucoup à la compréhension de l'économie et des sociétés urbaines occidentales par une mise en perspective du grand commerce de la soie, mais aussi du cas parisien avec celui des villes de Flandre et d'Italie. Autant le dire tout de suite : il s'agit d'une très belle leçon d'histoire, qui met en œuvre avec beaucoup de finesse et de pédagogie un matériel éclectique et dispersé.

2 Il s'agit donc, à partir du cas parisien, de mettre en évidence le système socioéconomique qui structure l'industrie de la soie. À l'origine, il y a cette fibre animale qui a transité par la Méditerranée et dont la technicité du travail comme les voies d'acheminement posent la question du rôle des intermédiaires dans son implantation à 
Paris, une des rares villes dotées d'une industrie de la soie à la fin du XIII ${ }^{\mathrm{e}}$ siècle, et la seule au nord des Alpes. C'est l'occasion pour l'auteure de repérer dans les rôles de taille deux cents contribuables d'origine méditerranéenne vers 1300, venant principalement de Lucques, Venise et Florence, mais aussi de la Péninsule ibérique, de Palestine, de Chypre, de Tunis et d'Alexandrie, avec une forte corrélation entre le métier exercé et les spécialités artisanales des pays d'origine qui confirme que l'industrie de la soie a dû naître d'une greffe extérieure.

Car cette fibre, si particulière par ses propriétés physiques, implique non seulement des compétences techniques qui expliquent le recours à des spécialistes étrangers, mais aussi une organisation du travail propre. La soie importée puis ouvrée à Paris est brute, mais une grosse moitié seulement des 1600 mètres de fils de chaque cocon est utilisable, si bien que le filage produit beaucoup de chutes de matière première. Par ailleurs, la gomme qui entoure les fils doit ensuite être dissoute par cuisson dans des bains d'eau pure, ce qui explique que les teinturiers de soie recherchent la proximité des fontaines acheminant l'eau de source, plutôt que celle de la Seine comme les teinturiers de laine. La soie est alors tissée au sein de corporations différentes: les tisserands de draps de soie et velours utilisent des métiers à tisser complexes produisant des bandes de tissu de cinquante-cinq centimètres environ, tandis que les tisserands de voiles, guimpes, couvre-chefs, bourses, ceintures et autres lacs de soie utilisent diverses techniques d'une moindre complexité pour produire des tresses, galons ou des bandes de tissu plus étroites. Tous les textiles mettant en œuvre la soie sont susceptibles d'être associés à des fils d'or produits par les orbateurs, et réciproquement.

4 Le coût de la matière première et ses caractéristiques techniques suscitent une organisation du travail particulière. La filière de la soie est dominée par les merciers, mais contrairement au cas italien, leur domination sur les autres acteurs de la filière est moins économique que juridique. Les merciers assez riches pour être entrepreneurs confient certes leur soie brute aux fileresses et aux fabricantes de couvre-chefs, qui tentent de leur soutirer de la matière première au passage en jouant sur le volume de bourre produit par leur activité, mais les teinturiers et les tisserands possèdent la soie qu'ils mettent en œuvre. Ils sont en revanche sous la surveillance des merciers qui, appartenant au milieu échevinal proche du roi, sont bien placés pour obtenir en 1324 du prévôt de Paris des droits de police sur toutes les corporations liées au travail de la soie. Il s'ensuit une concentration topographique des principales corporations de la filière autour des riches merciers entrepreneurs des rues Troussevache, Quincampoix et de la Buffeterie. S'y concentrent les ouvriers de la petite mercerie artisanale (tisserands de tissus de soie ainsi que tisserands de rubans et lacs de soie) comme de l'industrie du drap de soie, avec ses fileuses de soie, tisserandes de voiles, tisserands de draps de soie, velours et draps d'or, teinturiers, orbateurs et fileuses de fils d'or. Une partie de ces merciers entrepreneurs sont des migrants issus des régions d'importation de la soie et l'auteur déduit de la proximité topographique avec des travailleurs de la soie originaires des mêmes régions qu'ils les ont incités à migrer à Paris - à moins qu'ils y aient tous été invités par une autorité supérieure, par exemple un prince ou le roi, la question restant ouverte faute de preuve.

5 Mais la technique a aussi des incidences sur la division genrée du travail, avec une nette dominante féminine dans certaines corporations, voire une exclusivité comme pour les fileuses de soie et les tisserandes de voiles. Ce modèle genré se traduit par une inégalité systématique de revenus qui s'explique par une inégale complexité technologique des 
outils de production et des compétences pour les mettre en œuvre, par la visibilité du produit final, qui est elle-même fortement corrélée avec son prix et une clientèle aristocratique, et enfin par l'absence d'autonomie. Dans l'industrie de la soie, les femmes paient a minima deux fois moins d'impôts, utilisent des métiers à tisser simples quand les tisserands de velours, par exemple, travaillent sur un métier à tisser à double ensouples, vendent de petits articles de soie sans appellation d'origine, tandis que les draps de soie et les velours sont identifiés jusqu'en Suède comme venant «de Paris ». Par ailleurs, aucune corporation féminine ne mentionne l'élection de garde du métier par les ouvrières, et certaines ajoutent une dépendance économique à cette absence d'autonomie juridique.

6 Et en même temps, force est de reconnaître que les femmes de la filière de la soie sont surreprésentées dans les rôles d'impôt, ce qui prouve qu'elles en vivent mieux que leurs consœurs d'autres filières, en particulier celle du drap de laine. Les travailleuses de la soie sont aussi les seules à s'organiser en métier, faisant de Paris une des rares villes d'Occident dotées de corporations strictement féminines. Surtout, les femmes tiennent une place enviable dans les corporations mixtes : les mercières représentent $13 \%$ des membres du métier, mais paient une taxe moyenne légèrement supérieure à celles de leurs confrères, et sont en bonne place parmi les fournisseurs réguliers de la cour. Cette capacité à accumuler du capital à tous les stades de la production, que l'on n'observe ni dans les villes d'Italie ni dans celles de Flandre, s'explique par un droit parisien favorable aux veuves et aux filles, qui bénéficient d'un partage égalitaire de l'héritage. Par ailleurs, les règlements de corporations excluent rarement les femmes du travail, peut-être parce qu'ils ne sont pas promulgués par les bourgeois, comme en Flandre, mais par le roi, et que les échevins parisiens, dont un certain nombre sont merciers, sont d'autant moins enclins à brider les affaires de leur épouse mercière qu'ils font par ailleurs de belles carrières dans l'administration locale et royale, par définition inaccessibles aux femmes - leur masculinité est donc sauve!

7 Si la coutume parisienne permet aux femmes issues de familles un minimum aisées d'accumuler du capital, il n'en reste pas moins beaucoup d'ouvrières pauvres dans la filière de la soie, qui en sont réduites pour survivre à mettre en gage la soie que leur a confiée leur mercier. C'est l'occasion pour S. Farmer d'explorer de façon très sensible les rapports de crédit, de travail et de genre entre ces femmes et leurs créanciers qui s'avèrent surtout être des juifs et des Lombards, deux communautés d'étrangers également liées à la filière de la soie. Il en ressort que ces femmes s'adressent plus volontiers aux juifs qu'aux Italiens, non seulement parce que ceux-ci sont spécialisés dans les prêts d'un montant élevé, mais encore parce qu'ils vivent à Paris en compagnie d'hommes, comme des expatriés, et sont plus menaçants sexuellement que les prêteurs sur gage juifs qui vivent en famille dans la capitale. De fait, les femmes seules ne viennent presque jamais solliciter un prêt auprès d'un Lombard, tandis qu'elles forment 26 à $50 \%$ des débitrices des juifs et qu'un tiers des prêteurs juifs sont des femmes. Mais cette proximité avec les juifs se double pour les travailleuses de la soie de liens professionnels puisque les statuts des faiseresses de chapeaux d'or prévoient que des apprenties peuvent apprendre le métier auprès de juifs et qu'on trouve quelques exemples de juives converties qui exercent dans la filière, parfois au plus haut niveau. Ces compétences artisanales s'expliquent probablement par la nécessité de fabriquer des ornements liturgiques, mais aussi par les liens qu'ont conservés les communautés juives de la France du Nord avec celles de Méditerranée. 
8 Le système socio-économique de l'industrie de la soie si bien décrit par S. Farmer se met en place brusquement à Paris vers 1280 , connaît probablement son apogée vers 1400 avec les fastes de la cour de Charles VI, puis disparaît dans la première moitié du $\mathrm{xv}^{\mathrm{e}}$ siècle avec le schisme royal. Il y a donc une corrélation chronologique parfaite entre l'essor de la dépense curiale dans la capitale et celui de l'industrie de la draperie de soie qui pousse l'auteure à supposer que l'arrivée d'artisans italiens résulte de migrations au gré des mariages princiers en Méditerranée ou d'une entreprise volontariste de la part $\mathrm{du}$ roi pour répondre aux besoins de la cour. C'est possible, mais pourquoi ne pas envisager tout simplement l'attractivité du marché curial parisien, concomitante du déclin des foires de Champagne? Car l'industrie de la soie qui se met alors en place ne part pas de rien : il existe à Paris un artisanat de petits articles de fils de soie et d'or avant 1220 , tandis que la cour n'a pas encore investi la ville. Quelle serait alors sa clientèle, sinon le clergé pour ses vêtements liturgiques qui ne sont pratiquement jamais évoqués par S. Farmer? On touche là un angle mort du modèle, qu'on aurait aimé voir exploré avec la même minutie que ses autres aspects, mais c'est bien là la seule critique que l'on peut faire à une enquête qui, par ailleurs, apporte beaucoup de neuf sur les rapports de travail, de genre et d'altérité à Paris, la dimension intégratrice et émancipatrice de l'organisation corporative, ainsi que l'apport des artisans immigrés dans le développement économique du royaume de France... que l'historiographie française a tendance à occulter pour valoriser ses racines rurales, comme le note malicieusement l'auteure américaine de cette belle étude.

\section{AUTEURS}

\section{BORIS BOVE}

Université Paris 8 Vincennes-Saint-Denis, MéMo 